Original Research Paper

\title{
The Statistical Examination of Winning and Succeeding in Sports
}

\author{
Elisee Joseph \\ School of Business, Felician University, Rutherford, NJ, USA
}

Article history

Received: 14-01-2019

Revised: 22-04-2019

Accepted: 26-04-2019

Email: Josephe1626@students.felician.edu

\section{Introduction}

The legendary coach Wooden (2001) once said: "You can lose when you outscore somebody in a game and you can win when you're outscored". Virtually all competitive games that involves two distinct teams are determined by the final accumulated score. But in many instances, the score in most games does not explain the full story between two competitive teams that have completed a tangible game. Oftentimes, there are other strategic factors that play a key role in winning a competitive game than just total points. These strategies are implemented in practices, film sessions, scouting reports, pre-game and post-game analysis. In concrete practices, most coaches, sporting analysts, economists and business executives pay little attention to the scoreboard after the winning team has been determined.

This paper deals with the important issue of cooperative success in game theory. In this paper, this scholarship recognizes a linear coalition model that assesses a competitive team's performance that disregards the accumulation of the total score. The purpose of this paper is to cultivate a key theory that contributes to the total success of the game between competitive two sports teams that are simultaneously trying to win a game. Additionally, the purpose of this paper is to apply this theory towards the economically competitive environment between interdependent firms.

In the discussion of the rules and regulation of all competitive team sports, the coalition success model in each competitive game doesn't include any rule and regulatory modification at any given time. Therefore, the number of players allowed in a game along with all other rules and regulation in a timely regulated sporting event remains unscathed. However, this model provides an additional outlook besides the scoring outcome of the game. This model also provides a perspective that can enable competitive firms to measure the team's overall performance without completely paying attention to the scoring output of competitive matches.

\section{Literature Review}

Certain sports statisticians have cultivated various sports models that denote a particular player's impact on a team's throughout a given game. Other sports analysts have also discussed advanced metrics that explain the performance of both teams in a sporting event. In the 
sport of basketball, Hollinger (2002) was one of the prominent sports statisticians who has effectively created a statistical model that allows other analysts to determine a basketball player's efficient contributions to the success of a particular team. Hollinger's Player Efficiency Rating (PER) states that:

$$
\begin{aligned}
& u P E R=\frac{1}{M P} *\left(3 P+\frac{2}{3} A S T+\left[\left(2-\text { Factor } *\left(\frac{T m_{A S T}}{T M_{F G}}\right) * F G\right]\right.\right. \\
& +\left[\frac{1}{2} F T *\left(2-\frac{1}{3}\left(\frac{T m_{A S T}}{T M_{F G}}\right)\right]-[V O P-T O]\right. \\
& -[V O P * D R B P *(F G A-F G)] \\
& -[(0.44 * V O P) *(0.44+(0.56 * D R B P)) *(F T A-F T)] \\
& +[V O P *(1-D R B P) *(T R B-O R B)] \\
& +[V O P * D R B P * O R B] \\
& +[V O P * S T L]+[V O P * D R B P+B L K] \\
& -\left[P F *\left(\frac{\lg _{F T}}{\lg _{P F}}\right)-0.44\left(\frac{\lg _{F T A}}{\lg _{P F}}\right) * V O P\right]
\end{aligned}
$$

where:

$$
\begin{aligned}
& \text { factor }=\frac{2}{3}-\left[\frac{\frac{1}{2}\left(\frac{T m_{A S T}}{T M_{F G}}\right)}{2 *\left(\frac{T m_{A S T}}{T M_{F G}}\right)}\right] \\
& V O P=\left[\frac{\lg _{P T S}}{\lg _{F G A}-\lg _{O R B}+\lg _{T O}+0.44 * \lg _{F T A}}\right] \\
& D R B \%=\frac{\lg _{T R B}-\lg _{O R B}}{\lg _{T R B}}
\end{aligned}
$$

With:

$\mathrm{tm}$ The prefix, indicating of the team rather than of player

$\lg$ The prefix, indicating of the league rather than of player

$\min$ For a number of minutes played

$3 P \quad$ For a number of three-point field goals made

$F G$ For a number of field goals made

$F T$ For a number of free throws made

$V O P$ For the value of possession (but in reference to the league, in this instance)

$R B$ For a number of rebounds: $O R B$ for offensive, $D R B$ for defensive, $T R B$ for (total) combined, $R B P$ for a percentage of offensive or defensive

In other proposed metrics like "Wins produced", "Win Score" and "Plus-Minus", many scholars like Berri (1999), Berri and Schmidt (2010) and Berri et al. (2006; 2007) attempted to address the issue of the effective value of a particular player in a given basketball event. These scholars also addressed the limitations of linear-weight style models in basketball, making a valid argument that these metrics often overrate the offensive attributes of individual players while undermining defensive and true offensive efficiency. Nonetheless, these metrics are extremely powerful when factoring a basketball player's contributions to the team's success over the course of a given game. However, this model does not address the overall team's success in an independent game.

Lewis (2003) provided focus on a team's analytical and statistical performance in a baseball game. Hakes and Sauer (2006) extended Lewis' approach by providing additional empirical evidence that supports the claim that a baseball team's performance goes beyond the box score output. James $(1980 ; 1981 ; 1982 ; 1983)$ proposed a rather sophisticated model in baseball that is parallel to the model that is discussed in this paper. The Pythagorean Win- Loss Record simply states that:

$$
\begin{aligned}
& \text { Win Ratio } \%=\frac{\text { Runs Scored }^{2}}{\text { RunScored }^{2}+\text { Run Allowed }^{2}} \\
& =\frac{1}{1+\left[\frac{\text { Run Allowed }}{\text { RunScored }}\right]^{2}}
\end{aligned}
$$

In addition to the overall consideration of the culminating Pythagorean formula, Bill James devised a Game Score to determine the pitcher's strength and efficiency in a given baseball game. This pitcher's game score is denoted as:

$$
\begin{aligned}
& 50+[\text { Outs } * 1]+[\text { Innings completed after the 4th Inning }] \\
& +[\text { Strikeouts } * 1]-[\text { Hits } * 2]-[\text { Earned Runs } * 4] \\
& -[\text { Unearned Runs } * 2]-[\text { Walks } * 1]
\end{aligned}
$$

With the derived support of empirical data by Jones and Tappin (2005). Miller (2007), Rothman (2014), Hammond et al. (2013) and Miller et al. (2014), this Pythagorean formula provides an expected value for a baseball team's winning percentage based on the number of runs scored and a number of runs allowed. Through the structural sophistication of the Pythagorean Win-Loss Record in baseball, this theorem has been used in other sports. With theoretical justification through the Weibull probability distribution, Dayaratna and Miller (2013) applied this theorem in the sport of hockey such that:

$$
\text { Win Ratio } \%=\frac{(\text { Goals Scored }+0.5)^{\gamma}}{(\text { Goals Scored }+0.5)^{\gamma}+(\text { Goals Allowed }+0.5)^{\gamma}}
$$

Through controversial variations, Morey (1993) and Hollinger found that the Pythagorean Win-Loss Record works well in basketball such that: 


$$
\text { Win Ratio } \%=\frac{\text { Points Scored }^{\gamma}}{\text { Points Scored }^{\gamma}+\text { OpposingPoints Scored }^{\gamma}}
$$

where, $\gamma$ varies between 13.91 and 16.5.

Schatz (2003) proposed that the football application of the Pythagorean projection should include the number of games played such that:

$$
\text { Win Ratio } \%=\frac{\text { Points Scored }^{2.37}}{\text { Points Scored }^{2.37}+\text { OpposingPoints Scored }^{2.37}}
$$

The Pythagorean Win-Loss Record empirically correlates with how certain teams perform in a game. This formula can also provide intuitive and plausible assumptions of a game. However, the in-game sequence of the James' Pythagorean Win-Loss Record can't be simulated with complete play-by-play data. This limitation allows other researchers an opportunity to create distinctive models that can simulate a given game while illuminating the predictive characteristics of a successful team without the explicit use of a scoreboard.

\section{The Success Model}

In a competitive match, only two teams compete to win a given sporting game. At any given time of regulation, one team is playing on offense (attacking position) and the other team is playing defense preventing the other team from successfully scoring. Since both teams are continuously trying to score a goal or defend the goal, both teams are inevitably making potential mistakes. In other words, there is no perfect match where a team makes no mistakes.

The success model goes as follows:

All $O(x)=$ offensive function, $D(x)=$ defensive function and $E(x)=$ error functions are all continuous functions $\Delta t \rightarrow 0$ (the time of the game endsapproaching 0:00).

Let $v$ be a game between two competing teams where Team $A=f(x)$ and Team $B=g(x)$ :

$$
\begin{aligned}
& f(x)=\lim _{x \rightarrow \infty} \sum_{i=1}^{n}\left(\frac{O(x)_{A}+D(x)_{A}}{E(x)_{A}}\right)_{i} \\
& g(x)=\lim _{x \rightarrow \infty} \sum_{i=1}^{n}\left(\frac{O(x)_{B}+D(x)_{B}}{E(x)_{B}}\right)_{i}
\end{aligned}
$$

The team with a higher ratio, regardless of the score of this game, is the successful team. Through parity, the successful team before the game starts is indeterminate where both team's ration is $0 / 0$. As time elapses, the function of both teams fluctuates simultaneously. During the game, both teams will jockey for the winning position. More precisely, the game continuously contains a system of functions where $f(x)=$ Team $A$ and $g(x)=$ Team $B$. For example, if team $A$ is winning a game at a given time, the system of functions will be:

$$
0 \leq \lim _{x \rightarrow \infty} \sum_{i=1}^{n}\left(\frac{O(x)_{B}+D(x)_{B}}{E(x)_{B}}\right)_{i} \leq \lim _{x \rightarrow \infty} \sum_{i=1}^{n}\left(\frac{O(x)_{A}+D(x)_{A}}{E(x)_{A}}\right)_{i}
$$

If Team $B$ is winning the game the system of functions will be:

$$
0 \leq \lim _{x \rightarrow \infty} \sum_{i=1}^{n}\left(\frac{O(x)_{A}+D(x)_{A}}{E(x)_{A}}\right)_{i} \leq \lim _{x \rightarrow \infty} \sum_{i=1}^{n}\left(\frac{O(x)_{B}+D(x)_{B}}{E(x)_{B}}\right)_{i}
$$

At the end of the game (where $\Delta t \rightarrow 0$ ), the team who makes the most positive plays while minimizing their mistakes will have a higher ratio than the opposing team- becoming the successful team in the game. This model can be used in major team sports that contain a shot clock or a timer - increasing the pace of the game. These sports include (but not limited to): Soccer, basketball and American football.

Game periods vary depending on a given game. For example, a sport like American Football, Men's professional basketball, Women professional basketball and NCAA Women's Basketball game has at least 4 distinct periods in a regulated game. (Including possible overtime periods) While a sport like Men's and Women's Soccer and NCAA Men's Basketball contains at least 2 distinct periods in regulation (including possible overtime periods). At any given game, multiple potential overtime periods may be required to extend the game if the regulation period did not determine a clear winner.

When it comes to the topic of the basketball sport, both teams, regardless of the in-game situation, are guaranteed at least one statistical outcome per possession. These outcomes include a Successful field goal attempt, an unsuccessful Field Goal attempt, a successful free throw(s) Attempt, an unsuccessful free throw(s) attempt, an assist, an offensive/defensive rebound, a turnover, a steal, a block and a foul (personal, flagrant, shooting, technical). Therefore, the offense, defense and mistake functions contain the total statistical measure of each nonconsecutive category:

- $\quad O(x)=$ Offensive Rebounds $($ OREB $)+$ Assists $(A S T)$ $+2 *(2-$ Point Field Goals Made $)+3 *(3-P o i n t$ Field Goals Made) + Total Free Throws Made (FT)

- $\quad D(x)=$ Defensive Rebounds $(D R E B)+$ Steals $(S T L)$ + Blocks $(B L K)$

- $E(x)=$ Missed field goals + Missed Free Throw Shots + Total Fouls $(P F)+$ Total Turnovers $(T O)$ 
In the competitive sport of American football, both team, regardless of offense or defense situation, are guaranteed an outcome of at least one statistical outcome per play in a given possession. These outcomes include: A touchdown, a kickoff return, a punt return, a deflected punt attempt, a successful field goal, an unsuccessful field goal, a field goal return for a touchdown, an interception, a fumble, a complete pass attempt, an incomplete pass attempt, gain of yards, a touchback, a loss of yards, a positive rushing attempt, a negative rushing attempt, a tackle, a sack, a safety, two-point conversions, a penalty flag on an ensuing play and turnover on downs.

Assuming that the rules and regulations of the game stays the same, the offensive, defensive and error function contains the total statistical component of each non-consecutive category:

- $O(x)=$ Total Offensive Yards $+6 *($ Touchdown $)+$ $3 *($ Successful Field Goal $(s)+2 *($ Two Point Conversions) + Point after Touchdowns $+2 *$ First Downs + Complete pass attempts + Total Return Yards (Interceptions, Fumbles, Kick Returns and Punt Returns)

- $\quad D(x)=1 / 4 *$ Total Tackles (including solo tackles, sacks and tackles for a loss on yards) + $2 *($ Safeties forced $)+$ Block Kicks (Punts, PAT, Field Goals) $+2 *$ Turnovers forced (Interceptions or Recovered Fumbles)

- $E(x)=$ Incomplete pass attempts + Punts + 3*(Unsuccessful Field Goals $)+$ Unsuccessful Two Point Conversion Attempts $+2 *$ Total turnovers (Fumbles, Intersections, Turnover on Downs) + Blocked Kicks (Punts, PAT, Field Goals) + Penalties

In a competitive game of hockey, both competing teams, regardless of offense or defensive positions, are guaranteed an outcome of at least one statistical outcome. These outcomes include a goal, saves, an assist, shots on goal and penalties in minutes. Assuming that the rules and regulations of the game stay the same, the offensive, defensive and error function contains the total statistical measure of each non-consecutive category:

- $O(x)=2 *$ Goals $+2 *$ Assists $+2 *$ Successful Shootout Attempt

- $D(x)=$ Blocks + Saves

- $E(x)=2^{*}$ Goals allowed + Shots allowed on Goal $+2 *$ Penalties in Minutes + Unsuccessful Shootout Attempt
In a competitive game of soccer, both competing teams, regardless of offense or defensive positions, are guaranteed an outcome of at least one statistical outcome. These outcomes include a goal, an assist, a shot, a penalty kick, a shot on target, a foul, yellow card(s), red card(s), offside(s) and corner(s).

Assuming that the rules and regulations of the game stay the same, the offensive, defensive and error function contains the total statistical mesure of each nonconsecutive category:

- $\quad O(x)=2 *$ Goals + Corner Kick(s) $+2 *$ Successful Penalty Kick $(s)+1 / 2 *($ Shots $)+$ Shots on Goal + Possession \% with Ball (decimal)

- $D(x)=2^{*}($ Saves $)$

- $E(x)=$ Fouls + Yellow Cards $+2 *$ Red Card $(s)+$ $2 *$ Goals Allowed + Possession \% without Ball (decimal)

\section{Success Rate Model: No Time Limit}

Certain sports do not have an explicit time limit. One notable sport that does not have a time limit in baseball. Baseball contains a period limit of at least 9 innings, including potential extra innings. All competitive match with no time limit contains two teams that are competing to win a given sporting game. During the match with no time limit, one team is simultaneously playing on offense (attacking position) while the other team is playing defense - preventing the other team from successfully scoring.

In a competitive game of Baseball (where $t \rightarrow \infty$ ), Team $A$ is playing offense in one half of the inning while Team B is playing defense during the same portion of the period. During the second half of the inning period, the roles of offense and defense situation are reversed. In a given half of an inning, both teams are guaranteed at least one statistical outcome. These outcomes include Strike(s), Ball(s), Foul ball(s), base on balls (walks), Base hit(s), Runs (including home runs and grand slams), runs batted in, a stolen base(s), an out (strikeout, put out, a double play, a triple play, caught stealing), error(s), assist(s) and balk(s). Assuming that the rules and regulations of the game stay the same, the offensive, defensive and error function contains the total statistical measure of each non-consecutive category:

- $O(X)=4 *($ Runs $)+$ Base Hit $(s)+$ Base on Ball $(s)$

- $D(x)=\frac{\text { Assist }(s)}{3}+$ Putout $(s)$

- $E(X)=1+(\operatorname{Error}(s)+$ Hits allowed $+4 *($ Runs allowed $)+$ Walks allowed $)_{1}+\ldots+($ Error $(s)+$ Hits allowed $+4 *($ Runs allowed $)+$ Walks allowed $)_{i}$ 


\section{With $i=$ number of innings}

Coaches along with offensive and defensive strategists can apply various strategies that can enable their respective teams to win a competitive game. Throughout any given sports game, the coaches and strategists can implement slow-pace or fast pace strategies offensive plays to efficiently score in a short period of time. The team strategists can also defend their goal by implementing double teams, one on one and zone defense. Most of these offensive or defensive strategies can be key factors that directly relate to the team's success during the game.

These strategies can simultaneously impede the progress of the opposing team's potential to win the competitive game. Regardless of the sport that is played, each decision maker (coaches, strategists and players) in a coalition must anticipate the reaction of the opposing coalition in a competitive game. Through the existence of market competition or even internal competition, certain executive strategies for coalition success can also be applied in economics where one coalition that contains employees, customers investors and stakeholders must anticipate a reaction of rivals including direct and indirect competitors, other employees, other customers and other investors.

\section{Empirical Example/Design/Approach:}

This researcher used the NCAA Men's Basketball Sweet 16 game between Duke University and Syracuse University (played on March 23, 2018) as an example that puts this statistical model in action. ${ }^{1}$ In any basketball game, the success rate for both teams is denoted by this model:

$$
\begin{aligned}
& \text { Success Rate } \\
& =\frac{2(F G)+3(3 P T F G)+F T+R E B+A S T+S T L+B L K}{T O+P F+\text { Missed FGs }+ \text { Missed FT }}
\end{aligned}
$$

In this particular example, this researcher progressively simulated the course of this game. At the start of this basketball game, the success rate for both basketball programs is indeterminate. At tip-off, the success rate for both teams is undefined since the basketball game did not commence (Table 1).

With 17 minutes and 32 seconds left in the first half, Syracuse University scored 5 points while shooting 50\% $(1 / 2$; 2-Point FG) from the perimeter and $100 \%(1 / 1$;

\footnotetext{
${ }^{1}$ Syracuse v. Duke Team Statistics (March 23, 2018). ESPN.com. Retrieved from http://www.espn.com/mens-collegebasketball/matchup?gameId=401025881
}

3PFG) outside the perimeter. Syracuse University also grabbed 1 rebound and dished out 2 assists while turning the ball over once. Simultaneously, Duke University scored 4 points, shooting $66 \%$ from the perimeter $(2 / 3$; 2-Point FG) while grabbing 1 rebound, obtaining 1 assist, 1 steal, 1 personal foul and 1 turnover. When using the metrics for this segment of the game, the respective success rate for Syracuse University and Duke University were 4 and 2.33 (Table 2):

Syracuse University Success Rate $=\frac{2(1)+3(1)+1+2}{1+0+1}=\frac{8}{2}=4$

Duke University Suvvess Rate $=\frac{2(2)+1+1+1}{1+1+1}=\frac{7}{3} \approx 2.33$

With 3 minutes and 45 seconds left in the first half, Syracuse University scored 27 points while shooting $42.1 \%(8 / 19 ; 2 \mathrm{PFG})$ from the perimeter, $60 \%(3 / 5 ; 3-$ Point FG) outside the perimeter and $100 \%(2 / 2)$ from the free throw line. At that time, Syracuse University obtained a total of 17 team rebounds, 6 assists, 2 steals, 1 block, 8 turnovers and 9 team fouls. Simultaneously, Duke University scored 26 points, shooting $50 \%$ from the perimeter $(6 / 12 ; 2$-Point FG), $33 \%$ outside the perimeter $(2 / 6 ; 3$-Point FG) and $66 \%$ $(8 / 12)$ from the free throw line. Duke University also obtained a total of 8 rebounds, 5 assists, 3 steals, 4 team fouls and 4 turnovers. When using the metrics for this segment of the game, the respective success rate for Syracuse University and Duke University were 1.77 and 1.82 (Table 3):

$$
\begin{aligned}
& \text { Syracuse University Success Rate }=\frac{2(8)+3(3)+2+17+6+1+2}{13+0+8+9}=\frac{53}{30} \approx 1.77 \\
& \text { DukeUniversity Suvvess Rate }=\frac{2(6)+3(2)+8+8+5+3}{10+4+4+4}=\frac{40}{22} \approx 1.82
\end{aligned}
$$

Although Syracuse University led at this point of the game, the metric rate suggests that Duke University was indeed successful.

At halftime, Syracuse University scored 27 points while shooting $40 \% \quad(8 / 20 ; 2$-Point $F G)$ from the perimeter, $50 \%(3 / 6 ; 3$-Point FG) outside the perimeter and $100 \%(2 / 2)$ from the free throw line. By the intermission period, Syracuse University obtained a total of 19 team rebounds, 6 assists, 3 steals, 1 block, 11 turnovers and 10 team fouls. Duke University scored 34 points, shooting $46.67 \%$ from the perimeter $(7 / 15 ; 2-$ Point FG), 37.5\% outside the perimeter (3/8; 3-Point FG) and $68.75 \%(11 / 16)$ from the free throw line. Duke University also obtained a total of 12 rebounds, 6 assists, 
5 steals, 5 team fouls and 5 turnovers. When using the metrics for this segment of the game, the respective success rate for Syracuse University and Duke University were 1.65 and 2.05 (Table 4):

$$
\begin{aligned}
& \text { Syracuse University Success Rate }=\frac{2(8)+3(3)+2+19+6+1+3}{13+0+11+10}=\frac{56}{34} \approx 1.65 \\
& \text { Duke University Suvvess Rate }=\frac{2(7)+3(3)+11+12+6+5}{13+5+5+5}=\frac{57}{28} \approx 2.04
\end{aligned}
$$

With 2 minutes and 39 seconds left in the second half of this game, Syracuse University scored a total of 55 points while shooting $54.28 \%(19 / 35 ; 2$-Point FG) from the perimeter, $27.27 \%(3 / 11 ; 3-$ Point $F G)$ outside the perimeter and $61.54 \%(8 / 13)$ from the free throw line. Additionally, Syracuse University obtained a total of 33 team rebounds, 11 assists, 3 steals, 1 block, 16 turnovers and 15 team fouls. Meanwhile, Duke University scored 63 points, shooting $55.17 \%$ from the perimeter (16/29; 2-Point FG), 20\% outside the perimeter $(5 / 25$; 3-Point FG) and $69.57 \%(16 / 23)$ from the free throw line. Duke University also obtained a total of 33 rebounds, 13 assists, 8 steals, 2 blocks, 14 team fouls and 7 turnovers. When using the metrics for this segment of the game, the respective success rate for Syracuse University and Duke University were 1.63 and 1.95 (Table 5):

$$
\begin{aligned}
& \text { SyracuseUniversity Success Rate }=\frac{2(19)+3(3)+8+33+11+3+1}{24+8+16+15}=\frac{103}{63} \approx 1.63 \\
& \text { DukeUniversity Suvvess Rate }=\frac{2(16)+3(5)+16+33+13+8+2}{33+7+7+14}=\frac{119}{61} \approx 1095
\end{aligned}
$$

At the end of the game, Syracuse University scored a total of 65 points while shooting $49.0 \%$ (25/51; total FG) from the floor, $30.8 \%(4 / 13$; 3-Point FG) outside the perimeter and $64.7 \%(11 / 17)$ from the free throw line. Additionally, Syracuse University obtained a total of 33 team rebounds, 11 assists, 3 steals, 1 block, 16 turnovers and 15 team fouls. Meanwhile, Duke University scored 63 points, shooting $39.3 \%$ from the floor (22/56; total FG), $20 \%$ outside the perimeter $(5 / 25$; 3 -Point FG) and $71.4 \%(20 / 28)$ from the free throw line. Duke University also obtained a total of 33 rebounds, 13 assists, 8 steals, 2 blocks, 14 team fouls and 7 turnovers. When using the metrics at the end of the game, the respective success

\begin{tabular}{|c|c|c|c|c|c|c|c|c|c|c|c|c|c|c|}
\hline Team & FG & FGA & $3 \mathrm{PM}$ & 3PA & FT & FTA & TREB & AST & STL & BLK & TO & PF & PTS & SR \\
\hline Syr & 0 & 0 & 0 & 0 & 0 & 0 & 0 & 0 & 0 & 0 & 0 & 0 & 0 & Undef \\
\hline Duke & 0 & 0 & 0 & 0 & 0 & 0 & 0 & 0 & 0 & 0 & 0 & 0 & 0 & Undef \\
\hline
\end{tabular}
rate for Syracuse University and Duke University were 1.79 and 1.92 (Table 6).

\begin{tabular}{|c|c|c|c|c|c|c|c|c|c|c|c|c|c|c|}
\hline Team & FG & FGA & 3PM & 3PA & FT & FTA & TREB & AST & STL & BLK & TO & PF & PTS & SR \\
\hline Syr & 1 & 2 & 1 & 1 & 0 & 0 & 1 & 2 & 0 & 0 & 1 & 0 & 5 & 4 \\
\hline Duke & 2 & 3 & 0 & 0 & 0 & 0 & 1 & 1 & 1 & 0 & 1 & 1 & 4 & 2.33 \\
\hline
\end{tabular}

Table 1: Time- 20:00 (1st Half)

\begin{tabular}{|c|c|c|c|c|c|c|c|c|c|c|c|c|c|c|}
\hline Team & FG & FGA & $3 \mathrm{PM}$ & $3 \mathrm{PA}$ & FT & FTA & TREB & AST & STL & BLK & TO & PF & PTS & SR \\
\hline Syr & 8 & 19 & 3 & 5 & 2 & 2 & 17 & 6 & 2 & 1 & 8 & 9 & 27 & 1.77 \\
\hline Duke & 6 & 12 & 2 & 6 & 8 & 12 & 8 & 5 & 3 & 0 & 4 & 4 & 26 & 1.82 \\
\hline
\end{tabular}

Table 2: Time- 17:32 (1st Half)

Table 3: Time-3:45 (1st Half) ${ }^{+}$

\begin{tabular}{|c|c|c|c|c|c|c|c|c|c|c|c|c|c|c|}
\hline Team & $\mathrm{FG}$ & FGA & $3 \mathrm{PM}$ & 3PA & FT & FTA & TREB & AST & STL & BLK & TO & PF & PTS & SR \\
\hline Syr & 8 & 20 & 3 & 6 & 2 & 2 & 19 & 6 & 3 & 1 & 11 & 10 & 27 & 1.65 \\
\hline Duke & 7 & 15 & 3 & 8 & 11 & 16 & 12 & 6 & 5 & 0 & 5 & 5 & 34 & 2.04 \\
\hline
\end{tabular}

${ }^{+}$Although Syracuse University is leading, the success rate is lower than Duke University.

\begin{tabular}{|c|c|c|c|c|c|c|c|c|c|c|c|c|c|c|}
\hline Team & $\mathrm{FG}$ & FGA & $3 \mathrm{PM}$ & $3 \mathrm{PA}$ & FT & FTA & TREB & AST & STL & BLK & $\mathrm{TO}$ & $\mathrm{PF}$ & PTS & SR \\
\hline Syr & 19 & 35 & 3 & 11 & 8 & 13 & 33 & 11 & 3 & 1 & 16 & 15 & 55 & 1.63 \\
\hline Duke & 16 & 29 & 5 & 25 & 16 & 23 & 33 & 13 & 8 & 2 & 7 & 14 & 63 & 1.95 \\
\hline
\end{tabular}

Table 4: Halftime

\begin{tabular}{|c|c|c|c|c|c|c|c|c|c|c|c|c|c|c|}
\hline Team & $\mathrm{FG}$ & FGA & 3PM & 3PA & FT & FTA & TREB & AST & STL & BLK & TO & $\mathrm{PF}$ & PTS & SR \\
\hline Syr & 21 & 38 & 4 & 13 & 11 & 17 & 37 & 12 & 3 & 1 & 16 & 19 & 65 & 1.79 \\
\hline Duke & 17 & 30 & 5 & 26 & 20 & 28 & 33 & 13 & 8 & 2 & 7 & 16 & 69 & 1.92 \\
\hline
\end{tabular}

Table 5: Time -2:39 (2nd Half)

Table 6: Game Final (when $\Delta \mathrm{t} \rightarrow 0$ ) 
The success rate of both teams fluctuated throughout the game. At the beginning of this game, sports analyst can assume that Syracuse had a better start than Duke based on the score and the ratio of this game. As the game progresses, Duke made strategic adjustments to gain control of this game, causing a decrease in Syracuse's momentum. In a closely contested sporting event, the success rate proved to be independent of the actual scoreboard of the game. In the last 3 minutes of the first half, the Duke reflected a higher success rate than Syracuse University despite losing by 1 point. The converse relationship between the scoreboard and success rate output is routine in a competitive event. However, this relationship can cultivate a valid assumption that the successful team will most likely win the sporting event. Through the independent indication of the score of this game (Duke: 69 v. Syracuse: 65) outcome of this game shows that Duke University was indeed successful winners in this game.
Adding additional data points, the second figure provides a comprehensive summary of the Syracuse University v. Duke University Sweet 16 game as shown in Fig. 1. The time series analysis represents the overall success of both teams throughout the game. Using this success model, sports analysts can determine key trends in different periods of the game.

In this paper, this researcher applied standard statistical data from the NBA, NFL, MLB and NHL. Throughout the 2017-2018 season, a total of 5,200 games were sampled in this study. Furthermore, this candidate applies the statistical model in every respective game while testing the correlation between the success rate of each team and the scoreboard outcome in each particular game.

\section{Empirical Results}

Table 7 highlights the distribution of each game within the season of the respective sports leagues. All statistics were retrieved from Sports Reference.

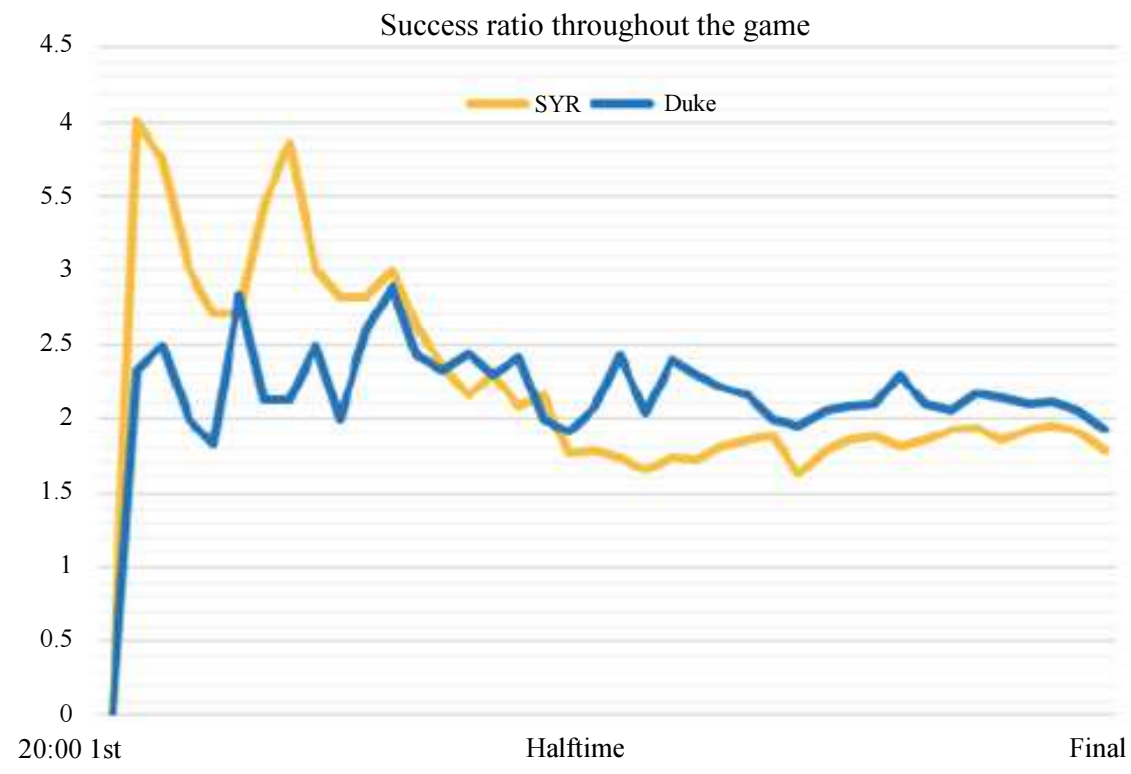

Fig. 1: Line graph of the game simulation at infinitely many different sequences

Table 7: The relationship between the success rate between winning teams of every game

\begin{tabular}{|c|c|c|c|c|c|c|}
\hline $\begin{array}{l}\text { Sports } \\
\text { league }\end{array}$ & Season & $\begin{array}{l}\text { Number } \\
\text { of games }\end{array}$ & $\begin{array}{l}\text { Unsuccessful } \\
\text { scoreboard winners** }\end{array}$ & $\begin{array}{l}\% \text { of Success } \\
\text { ratio agreement } * * *\end{array}$ & $\begin{array}{l}\text { Average } \\
\text { success ratio }\end{array}$ & $\begin{array}{l}\text { Average } \\
\text { scoreboard outcome }\end{array}$ \\
\hline MLB & 2017 Season & 2,430 & 52 & $98 \%$ & 2.52 & 4.65 \\
\hline $\mathrm{NBA}$ & 2017-2018 Season & 1,231 & 116 & $91 \%$ & 2.30 & 106.38 \\
\hline NFL & 2017-2018 Season* & 268 & 38 & $86 \%$ & 21.25 & 21.86 \\
\hline NHL & 2017-2018 Season & 1,271 & 100 & $92 \%$ & 0.86 & 2.97 \\
\hline Total & 5,200 & 306 & $\sim 94 \%$ & - & - & \\
\hline
\end{tabular}

*Including playoff games

** Unsuccessful scoreboard winners reflect a particular team that has won a game with a lower success rate than the losing team.

$* * *$ The success rate reflects the percentages of games where the winning team has a higher success rate than the losing team. 
Results suggest that there is a $94 \%$ correlation between the successes of the winning teams throughout the 2017-2018 seasons. Conversely, there were 306 events that contain contradictory results where the winning teams had a lower success rate than the losing team. This statistical model redefines John Wooden's distinction between winning and succeeding (Irvine, 2009). Throughout his TED talk in 2001, John Wooden provided a profound idea that a team "can still lose a game when they outscore the losing team and the losing team can still win a game when they are outscored". This statistical model suggests that a scoreboard does not portray the entire story of a particular game. The results, however, highlights certain plays and actions that took place in every possession, while demonstrating whether or not the team was generally successful throughout the game in other areas besides scoring.

\section{Conclusion}

This paper shows that the overall summary of a competitive match does not entirely depend on the outcome score of a game. This researcher used a statistical model that determines a team or a firm's overall success (and possible failure) within a given period of time. Regardless of time restrictions, the model can be used in any sport that comprises of a competitive match between two opposing teams. Although this theory can be applied without the explicit acknowledgment of a game score, this model does not alter the rules and regulations of any sport.

Additionally, this model does not include advanced statistics across all sports. For example, the sport of basketball has standard statistics like Points, Rebounds, Assist, Turnovers, Steals, Blocks and fouls. These standard categorical statistics are included in the model. However, this model excludes advanced metrics like player impact estimation, offensive and defensive ratings, Net rating, assists percentages, true shooting percentages, pace and player efficiency ratings. Though this model excludes advanced metrics, this model provides a different perspective that determines the success or failure of a sports team and firms without the fixation of a scoreboard.

This model can be applied to sports betting. This fundamental principle of this model can be also applied in economics and finance. In economics, all forms of resources including capital, land, labor and time are scarce. Therefore, market participants have to strategically find ways to allocate these resources and satisfy the wants and needs of both producers and consumers. In game theory, market competition arises because a plethora of participants is contending to obtain resources in an efficient manner. This form of competition leads to a zero-sum game where a participant's gain in resources may result in its competitor's loss in resources. In this statistical model, the activities of the successful team will ultimately affect the opposing team's chances of succeeding in this game.

In finance, the graphical characteristics of this statistical model relate to the principles of technical analysis in financial stock charts. Stock charts display key market trends over time. These trends can provide information pertaining to the market conditions for an index or a particular condition of a company's stock price. This statistical model also demonstrates certain trends throughout the game. For future research, this research plans on speculating a possible correlation between the results of a sporting event and the outcome of the financial markets over time.

\section{Ethics}

This article is original and contains unpublished material. The corresponding author confirms that all of the other authors have read and approved the manuscript and no ethical issues involved.

\section{References}

Berri, D.J. and M.B. Schmidt, 2010. Stumbling on wins: Two economists expose the pitfallson the road to victory in professional sports. Stanford Business Books.

Berri, D.J., 1999. Who is most valuable? Measuring the player's production of wins in the national basketball association. Managerial Decis. Econom., 20: 411427.

Berri, D.J., M.B. Schmidt and S.L. Brook, 2006. The wages of wins: Taking measure of the many myths in modern sport. Stanford University Press.

Berri, D.J., S. Brook and N. Schmidt, 2007. Does one simply need to score to score? Int. J. Sports Finance, 2: 190-205.

Dayaratna, K. and S. Miller, 2013. The Pythagorean won-loss formula and hockey: A statistical justification for using the classic baseball formula as an evaluative tool in hockey. Hockey Res. J., 16: 193-209.

Hakes, J.K. and R.D. Sauer, 2006. An economic evaluation of the Moneyball hypothesis. J. Economic Perspectives, 20: 173-185.

Hammond, C., W. Johnson and S. Miller, 2013. The James function.

Hollinger, J., 2002. Pro Basketball Prospectus. Beasley Sports, Washington D.C.

Irvine, J., 2009. John wooden on teaching. Bleacher Report.

James, B., 1980. Baseball abstract. Self-Published, Lawrence, KS.

James, B., 1981. Baseball abstract. Self-published, Lawrence, KS. 
James, B., 1982. Baseball abstract. Ballantine, New York. James, B., 1983. Baseball abstract. Ballantine, New York.

Jones, M. and L. Tappin, 2005. The Pythagorean theorem of baseball and alternative models. UMAP J.

Lewis, M., 2003. Moneyball: The Art of Winning an Unfair Game. W.W. Norton and Company, New York.

Miller, S., 2007. A derivation of the Pythagorean won-loss formula in baseball. Chance Magazine, 1: 40-48.

Miller, S., T. Corcoran, J. Gossels, V. Luo and J. Porfilio, 2014. Pythagoras at the Bat. In: Social Networks and the Economics of Sports, Pardalos, P. and V.A. Zamaraev (Eds.), Springer-Verlag, Berlin, pp: 89-113.
Morey, D., 1993. Daryl Morey's modified Pythagorean Theorem. STATS Basketball Scoreboard.

Rothman, S., 2014. A new formula to predict a team's winning percentage. Baseball Res. J., 43: 97-105.

Schatz, A., 2003. Pythagoras on the Gridiron. Football Outsiders.

Wooden, J., 2001. The difference between winning and succeeding. 